\title{
Physical meaning of the natural orbitals: Analysis of exactly solvable models
}

\author{
N. Helbig, ${ }^{1}$ I. V. Tokatly, ${ }^{1,2}$ and A. Rubio ${ }^{1,3}$ \\ ${ }^{1}$ Nano-Bio Spectroscopy Group and ETSF Scientific Development Centre, Departamento Física de Materiales, Universidad del País Vasco, \\ Centro de Física de Materiales CSIC-UPV/EHU-MPC and DIPC, Av. Tolosa 72, E-20018 San Sebastián, Spain \\ ${ }^{2}$ IKERBASQUE, Basque Foundation for Science, E-48011 Bilbao, Spain \\ ${ }^{3}$ Fritz-Haber-Institut der Max-Planck-Gesellschaft, Berlin, Germany
}

(Received 13 October 2009; published 9 February 2010)

\begin{abstract}
We investigate the suitability of natural orbitals as a basis for describing many-body excitations. We analyze to which extent the natural orbitals describe both bound as well as ionized excited states and show that depending on the specifics of the excited state the ground-state natural orbitals may yield a good approximation. We show that the success of reduced density-matrix functional theory in describing molecular dissociation lies in the flexibility provided by fractional occupation numbers while the role of the natural orbitals is minor.
\end{abstract}

DOI: 10.1103/PhysRevA.81.022504

PACS number(s): 31.15.X-, 31.15.ac

Density functional theory (DFT) $[1,2]$ has become one of the most widely used tools in electronic structure calculations. However, several problems remain that cannot be adequately described with the available DFT functionals. For one of these situations, the dissociation of small molecules, reduced density matrix functional theory (RDMFT) has shown promising results [3,4]. RDMFT is based on a one-to-one correspondence between the ground-state one-body density matrix (1-RDM) and the ground-state many-body wave function. This oneto-one mapping was proven by Gilbert in 1975 [5]. Several functionals of the 1-RDM have appeared over the years, most of them being functionals of the natural orbitals and occupation numbers, i.e., the eigenfunctions and eigenvalues of the 1-RDM, rather than the 1-RDM itself [3,4,6-14].

The promising results obtained with 1-RDM functionals have fueled a discussion of the physical meaning of the natural orbitals. Due to the fractional nature of the occupation numbers the physical significance of the natural orbitals is not obvious. They are defined as the eigenfunctions of the 1-RDM, i.e., as purely mathematical objects. Experience, however, shows that they are more often than not very close to the Hartree-Fock (HF) orbitals of a system. In other words, they seem to contain some physical significance as the HF orbitals are known to do, for example from Koopman's theorem. The similarity between the $\mathrm{HF}$ and natural orbitals is especially striking for the homogeneous electron gas, where the natural orbitals are plane waves due to symmetry and, hence, identical to the HF orbitals. For the occupation numbers one obtains a smoothed step function with reduced step size instead of the perfect step function of HF. Generally, if one can show that the natural orbitals resemble single particle orbitals one can connect them to single particle energies and, hence, obtain a single-particle spectrum as an approximation to the true spectrum of a system. Therefore, it is important to answer the question of the physical meaning contained in the natural orbitals. Unfortunately, most RDMFT calculations minimize the total energy which contains a small part that is only known approximately. Consequently, one does not obtain the true natural orbitals of the system but some approximate ones and, hence, cannot distinguish whether a nonphysical behavior of the orbital is real or just a result of the approximation. Therefore, it is necessary to investigate the natural orbitals for a system where one has access to the exact 1-RDM.

In this paper we investigate the natural orbitals for several model systems. We choose one-dimensional (1D) two-electron systems because they are mathematically identical to a oneparticle system in two dimensions. Hence, the exact twoparticle wave function can be obtained numerically, and the exact 1-RDM and natural orbitals can be calculated. As a first system we choose a single potential well and different interaction strength between the two electrons. We investigate the natural orbitals not only for the ground state but also for the first excited state in order to see if two-particle excitations can be described by only changing the occupation numbers of the ground-state natural orbitals. The second system, two wells separated by an adjustable distance, allows us to discuss why RDMFT yields very good results in the dissociation of molecules. Here, we focus on the change in occupation numbers with increasing distance, i.e., with increasing correlation. Both models allow us to smoothly increase the correlation in the system by changing the interaction strength or the distance, respectively. Also, they are chosen such that we can investigate both the strongly and weakly correlated regimes in both cases. While the first model yields a correlation between a localized and a delocalized state, the second describes the correlation between two states localized in different parts of space. Hence, the two models correspond to two prototypical examples of strong correlation: Kondo physics and Hubbard correlation.

The paper is structured as follows. First, we briefly introduce the basics of RDMFT and fix our notation. We then discuss the single well system and the question if the natural orbitals are suitable for describing many-particle excitations. We focus on the dissociation of a two-well system before we conclude our findings.

\section{THEORETICAL DESCRIPTION}

The one-body density matrix of a system is calculated from its wave function via

$$
\begin{aligned}
\gamma_{k}\left(\mathbf{r}, \mathbf{r}^{\prime}\right)= & N \int d^{3} r_{2} \cdots d^{3} r_{N} \Psi_{k}^{*}\left(\mathbf{r}^{\prime}, \mathbf{r}_{2}, \ldots, \mathbf{r}_{N}\right) \\
& \times \Psi_{k}\left(\mathbf{r}, \mathbf{r}_{2}, \ldots, \mathbf{r}_{N}\right),
\end{aligned}
$$


where $\Psi_{k}$ is the $N$-electron wave function. For $k=0$ one obtains the ground-state density matrix which serves as the fundamental variable in RDMFT. Equation (1) can easily be modified to include spin. However, since we only discuss two electron systems in this work, the spin and spatial variables separate and spin is implicitly included in the symmetry of the spatial wave function. Instead of the 1-RDM one can employ its eigenvalues and eigenfunctions obtained from

$$
\int d^{3} r^{\prime} \gamma_{0}\left(\mathbf{r}, \mathbf{r}^{\prime}\right) \varphi_{j}\left(\mathbf{r}^{\prime}\right)=n_{j} \varphi_{j}(\mathbf{r})
$$

The eigenfunctions $\varphi_{j}$ are known as natural orbitals with the eigenvalues $n_{j}$ being their occupation numbers. The natural orbitals and occupation numbers for excited states can be obtained in analogy to Eq. (2). In order for a 1-RDM to be $N$-representable the occupation numbers have to fulfill two conditions [15], namely,

$$
\sum_{j=1}^{\infty} n_{j}=N, \quad 0 \leqslant n_{j} \leqslant 1 .
$$

For noninteracting electrons the occupation numbers are strictly 0 or 1 while for interacting electrons some, if not all, occupation numbers are fractional. In case the system is closed-shell the natural orbitals of the two spin directions are identical. As a result, we can choose to work with half the number of natural orbitals and occupation numbers between zero and two. We have made that choice for all the closed-shell systems presented here.

Since one of the goals of RDMFT is the description of strongly correlated systems, we also define the correlation entropy [16]

$$
s=-\frac{1}{N} \operatorname{tr}(\gamma \ln \gamma)=-\frac{1}{N} \sum_{j=1}^{\infty} n_{j} \ln n_{j},
$$

where tr denotes a trace. The correlation entropy describes the entanglement of the $N-1$ variables, that were traced out in the calculation of $\gamma$, and the remaining variable. In other words, it is a measure of the entanglement between one particle and the other $N-1$ particles in the system. For noninteracting particles, where the occupation numbers are strictly zero and one only, the correlation entropy is zero. A maximum contribution is obtained for $n_{j}=e^{-1}$ but the case where all occupation numbers obtain this value is usually not compatible with the total number of particles. Hence, as we will see in the following, the signature of strong correlation is half-occupation for the natural orbitals. For closed-shell systems, if one chooses to work with occupation numbers between zero and two, each $n_{j}$ needs to be divided by two and the whole sum multiplied by two, for the spin summation, in order to obtain the correct correlation entropy.

We consider 1D two-electron systems, hence, the Hamiltonian is given by (atomic units are used throughout)

$$
-\frac{d^{2}}{2 d x_{1}^{2}}-\frac{d^{2}}{2 d x_{2}^{2}}+v_{\text {ext }}\left(x_{1}\right)+v_{\text {ext }}\left(x_{2}\right)+v_{\text {int }}\left(\left|x_{1}-x_{2}\right|\right),
$$

where $v_{\text {ext }}$ denotes the external potential and $v_{\text {int }}$ the electronelectron interaction. As we can see, the Hamiltonian is mathematically equivalent to a single electron in two-dimensions with the $2 \mathrm{D}$ external potential

$$
v_{\text {ext }}^{2 \mathrm{D}}\left(x_{1}, x_{2}\right)=v_{\text {ext }}\left(x_{1}\right)+v_{\text {ext }}\left(x_{2}\right)+v_{\text {int }}\left(\left|x_{1}-x_{2}\right|\right) \text {. }
$$

The wave function for this problem can be calculated with any numerical code that can treat noninteracting electrons in 2D. The 1-RDM and the natural orbitals and occupation numbers can then be obtained via Eqs. (1) and (2). We use the OCTOPUS code $[17,18]$ for all the calculations presented here. The calculations were performed in a finite box with zero boundary conditions. Also, we consider systems at zero temperature as reflected in the Hamiltonian, Eq. (5).

\section{DESCRIPTION OF EXCITATIONS}

We consider a single potential well of hyperbolic cosine form, i.e., the external potential is given by

$$
v_{\text {ext }}(x)=-\frac{v}{\cosh ^{2}(\kappa x)} .
$$

The single particle eigenvalues of the system are given by [19]

$$
\epsilon_{n}=-\frac{\kappa^{2}}{8}\left[\sqrt{1+\frac{8 v}{\kappa^{2}}}-1-2 n\right]^{2}
$$

where it is understood that the square bracket needs to be positive. Hence, for $v / \kappa^{2}<1$ there exist only a single bound state while for $1<v / \kappa^{2}<3$ there are exactly two bound states for noninteracting particles. In our discussion of the physical interpretation of the natural orbitals we consider two scenarios: In both cases we choose $\kappa=1$, but $v=0.9$ in the first and $v=2.0$ in the second case. While the situation with exactly one bound state seems rather artificial for quantum chemistry it is frequently encountered in semiconductor nanostructures or in metals in the context of the Kondo effect and Anderson impurities, to name two examples. For the electron-electron interaction we choose a finite-range interaction, namely,

$$
v_{\text {int }}(x)=-\frac{b}{\cosh ^{2}\left(x_{1}-x_{2}\right)}
$$

with a variable interaction strength $b$. The external potential, Eq. (7) is symmetric and, therefore, the two-particle wave function can be chosen as an eigenfunction of the parity operator. As a result, the 1-RDM is symmetric under parity and the natural orbitals are simultaneous eigenfunctions of the 1-RDM and the parity operator. Consequently, we can order them with increasing number of nodes, i.e., starting with a nodeless symmetric natural orbital followed by an antisymmetric one with one node and so on. Alternatively, one can order the natural orbitals with decreasing occupation, i.e., the first natural orbital is the one with the highest occupation. The two orders do not necessarily coincide, as one of our examples shows. Throughout this paper we have chosen to order the natural orbitals according to their occupation number.

If there is only one bound state, the two noninteracting electrons occupy this state in a singlet configuration. However, if we continuously increase the interaction between the two electrons the energy of this state increases and eventually the two-electron wave function resembles one electron in the bound state and the other in an extended state, i.e., the system ionizes. If the system contains two bound states then 
the increase in the interaction strength also leads to an increase in the energy of the noninteracting ground-state configuration, however, the new ground state is still localized, i.e., the system does not ionize due to the existence of the second bound state. Of course, upon further increase of the interaction this system also ionizes but only at an interaction strength significantly higher than for the system with only one bound state. In the following we discuss the behavior of the natural orbitals for different interaction strength $b$.

In order to answer the question whether an excitation of the many-body system can be described by the natural orbitals of the ground state we investigate them for the ground- and the first excited states. If the natural orbitals of these two states are indeed very similar then the excitation can be described by a change in the occupation numbers alone. As a result, the energy spectrum of the many-body system could be obtained from assigning single-particle energies to the natural orbitals and calculating the appropriate weighted sum to obtain the manybody energy. All calculations in this section were performed in a box ranging from -15 to 15 with a spacing of 0.05 .

Figure 1 shows the first three natural orbitals of the ground state for an external potential with $v=0.9$. For noninteracting electrons, i.e., $b=0.0$, only the first orbital is occupied while all other orbitals are empty. The empty orbitals are numerically not accessible from a diagonalization of the density matrix and, since they are all degenerate with occupation number zero, they are only defined up to unitary transformations in the degenerate subspace. The most natural choice is, of course, the single particle eigenstates of the problem, which is what we plotted in Fig. 1 for $b=0.0$. As we can see, the orbitals are essentially the eigenstates of the box, i.e., cos and sin functions, with a small modification at the position of the potential well. A tiny interaction, $b=0.01$, leaves the first natural orbital unchanged but, as we can see in Fig. 1, the higher natural orbitals become very localized. This holds true for all the natural orbitals that
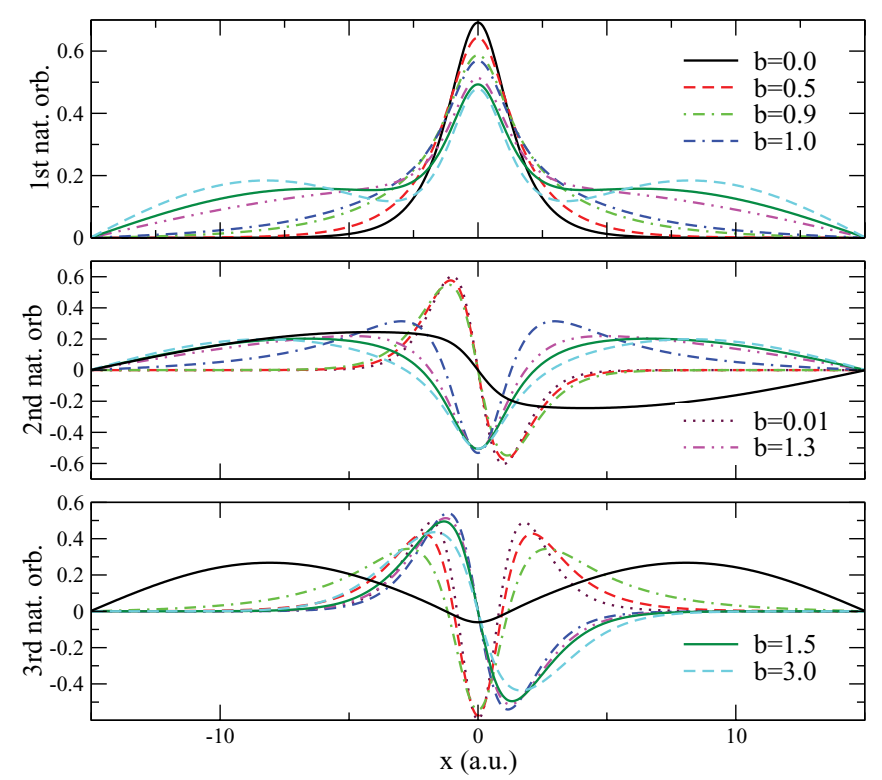

FIG. 1. (Color online) First, second, and third natural orbital of the ground-state density matrix for different interaction strength. For noninteracting electrons the second and third natural orbital are extended but immediately localize when the interaction is turned on.

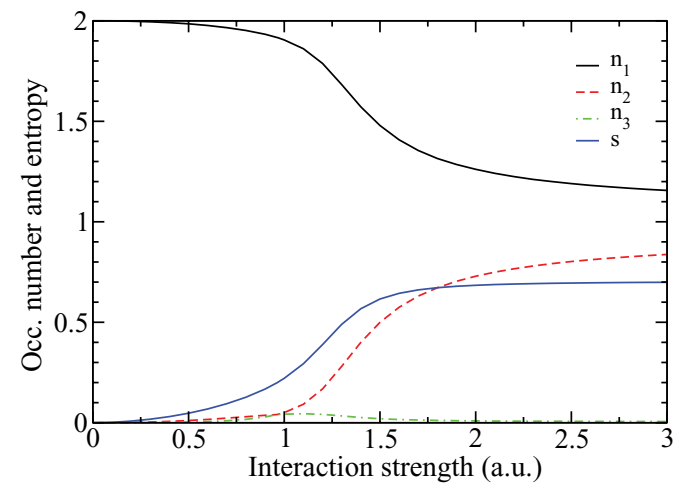

FIG. 2. (Color online) Three largest occupation numbers of the ground-state density matrix and correlation entropy, $s$, as a function of the interaction strength $b$.

we included in our calculation, not only these two. At first this is surprising since the small interaction represents a small perturbation of the noninteracting system. However, for the natural orbitals this perturbation acts on a highly degenerate subspace, all natural orbitals previously had zero occupation. Hence, all the extended states mix and form localized orbitals. Consequently, even an infinitesimal interaction leads to the whole set of natural orbitals to localize in the potential well. Since the occupation number of the first natural orbital is still almost 2 and all remaining occupation numbers are very small, see Fig. 2, the 1-RDM is almost identical to the one for noninteracting electrons, confirming that the perturbation is indeed small.

From Fig. 1 we see that around $b=0.9$ the shape of the second natural orbital changes quite dramatically from an orbitals with one node to one with two nodes. However, a comparison with the third natural orbital reveals that this natural orbital undergoes the opposite transition. Hence, it seems that the two orbitals have switched places which is confirmed by a look at the occupation numbers in Fig. 2 which are identical for $b$ slightly above 0.9 . In other words, the drastic changes in the second and third natural orbitals are a result of the fact that we always order them with decreasing occupation number. If we had ordered them according to their parity, each natural orbital would show a smooth change along the whole range of $b$ and the occupation numbers in Fig. 2 would cross.

All three natural orbitals show an increasing delocalized part above $b=1.0$. Again, since we are running the simulations in a finite box, these extended states resemble the eigenstates of the box. We have ensured that the box is large enough for the localized parts of the orbitals to remain unaffected by a change in the size of the box. However, the extended parts of course depend on the choice of box size. We interpret these results as an increase in the degree of ionization of the ground state of the system. In other words, the two electrons do not occupy the same single-particle state, i.e., the one bound state of the system, anymore. Due to the increased interaction one of the electrons is forced to occupy a different level, and since no other bound state is available it occupies an extended state. Of course, this only approximately describes the situation since the two electrons are interacting and, hence, the notion of single-particle levels is not totally appropriate. The occupation numbers, Fig. 2, 

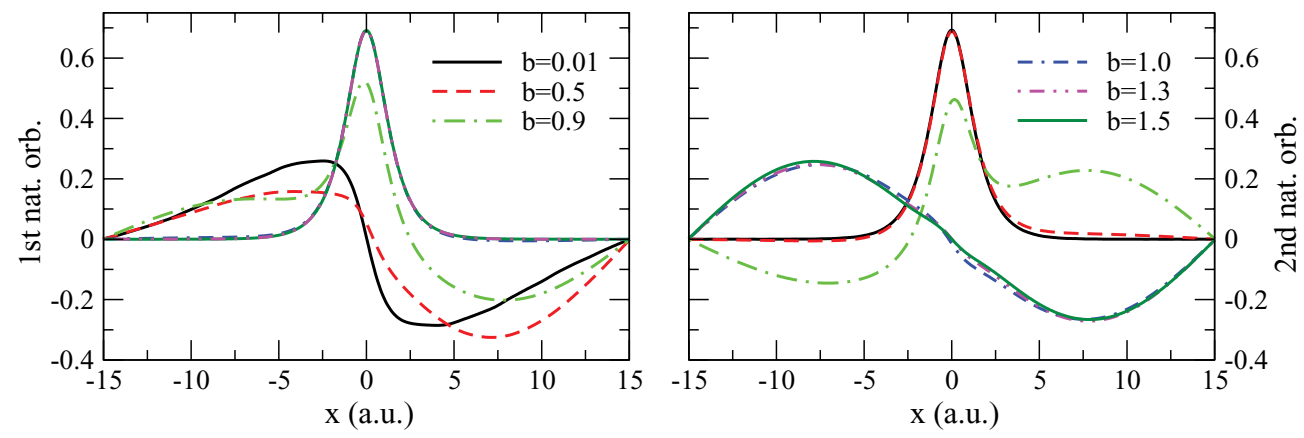

FIG. 3. (Color online) First and second natural orbital of the density matrix of the first excited state, a spin triplet, for different interaction strength. For $b=0.0$ the first two excited two-particle states are degenerate leading to an ambiguity in the natural orbitals. A small interaction, $b=0.01$ lifts the degeneracy and yields the results shown here.

show the increase in interaction by deviating more and more from their noninteracting values, zero and two. For $b \rightarrow \infty$ the two largest occupation numbers approach one and are degenerate. Looking at the evolution of the first two natural orbitals for $b>1.0$ it is clear that one can form a linear combination with one natural orbital completely localized while the other represents a slightly modified box states. Physically this describes the situation at infinite interaction strength where the ground state contains one localized and one completely delocalized electron. We also note that the two degenerate natural orbitals are both of even parity. Figure 2 also shows that the correlation entropy increases with increasing interaction strength and converges to a value of about 0.7 . In other words, for large interaction the situation closely resembles what is usually described as Kondo physics: the strong correlation between a localized and a delocalized state.

The first two natural orbitals for the first excited state of our two-electron system are plotted in Fig. 3. Since the first excited state is a spin triplet the excitation contains a spin flip of one of the two electrons. As we can see, the first natural orbitals is delocalized for noninteracting electrons but becomes localized between $b=0.9$ and $b=1.0$. The second natural orbital shows the opposite trend. The change is due to the fact that for a triplet state all occupation numbers are doubly degenerate. As a result, the natural orbitals are only determined up to a rotation in the degenerate subspace which implies that they are not necessarily parity eigenstates. One striking result, however, is the fact that over the whole range of interactions, one of the two natural orbitals is delocalized. This is not surprising as we expect the first excited state of a system with only one bound state to be partially ionized. As a result, this excitation cannot be described by changing the occupation of the ground-state natural orbitals at least in the range of $b<1.0$, where all ground-state natural orbitals are very well localized. In other words, while a description of many-body excitations via single particle excitations is no problem for noninteracting electrons, for a small interaction the natural orbitals are not suited for such a description. We note that for noninteracting electrons the first and second excited two-particle states are degenerate. As a result, any linear combination of the two states is an eigenfunction of the Hamiltonian leading to an ambiguity of the natural orbitals for the first excited state. We avoid this effect by plotting the natural orbitals for a very small interaction of $b=0.01$ which is sufficient to lift the degeneracy.

The inability of the natural orbitals to describe the excitation above is likely a result of the fact that our system has only one bound state and, hence, any excitation involves an ionization. Therefore, we increase the depth of our well by choosing $v=$ 2.0 which results in a second bound state for the noninteracting electrons.

Figure 4 shows the first and second natural orbital of the ground and first-excited states of the system with $v=2.0$. As we can see, over the whole range of interaction the natural orbitals of the ground state hardly change at all. Due to the much deeper well the first two natural orbitals stay well localized within the well even for relatively large interaction strength. Also, the change in the first natural orbital of the first excited state is almost unnoticeable on the scale of the plot. The second natural orbital, however, shows quite
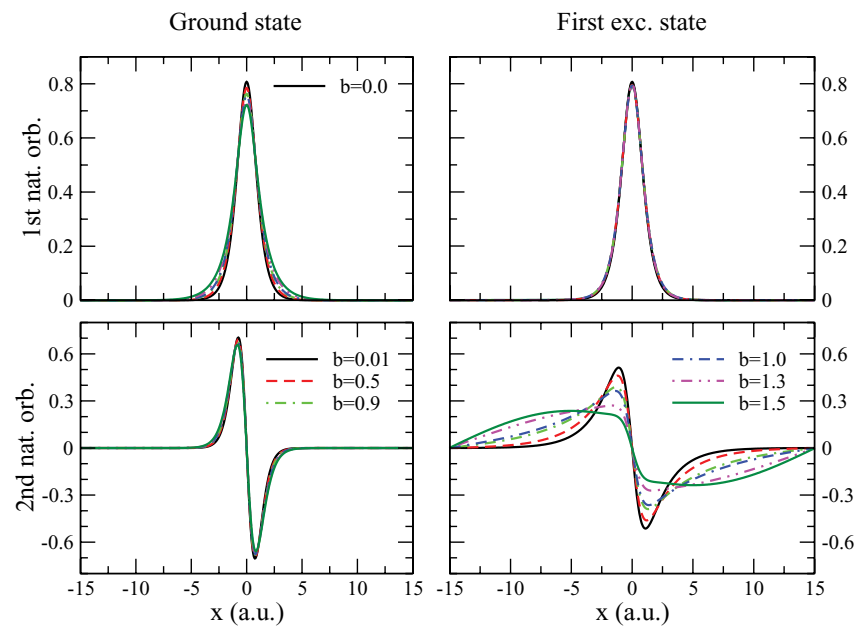

FIG. 4. (Color online) First and second natural orbitals of the ground-state density matrix (left) and the density matrix of the first excited state (right) for different interaction strength for a potential with $v=2.0$. The ground state is a spin singlet while the first excited state is a triplet. All graphs share the same color coding unless otherwise stated. For $b=0.0$ the second natural orbital of the ground state is numerically ill defined. Also, the same degeneracy of the first two excited states as before is encountered for noninteracting electrons. 
a pronounced change with increasing interaction strength. It becomes increasingly more delocalized with larger interaction strength and obtains features of the box state similar to the behavior of the natural orbitals of the ground state for the smaller potential well, see Fig. 1. This can be understood if one keeps in mind that the energy of the excited state is closer to the continuum and, therefore, acquires a certain degree of ionization much earlier than the ground state. We also observe that the form of the two natural orbitals, especially for small interaction strength, is similar for the ground and the first-excited state. Looking at the occupation numbers we notice that the first two orbitals for the excited state are equally occupied with an occupation of 1.000 at $b=0.01$ decreasing to 0.998 at $b=1.5$. For the ground state the occupation of the first natural orbital decreases from 2.000 to 1.957 , respectively. Consequently, removing one electron from the first natural orbital of the ground state and placing it into the second one yields a very good description of the first excited state for small interaction strength and is expected to still be a reasonably good approximation for intermediate $b$.

Our calculations suggest that excitations between manybody states that are of similar nature, for example two well-localized bound states, can be described by only changing the occupation numbers of the ground-state natural orbitals. Any excitations that involve an ionization of the system, however, cannot be expected to be well described by using the ground-state natural orbitals. Also, an excitation from a localized low-energy state to a high-energy Rydberg state in an atom cannot be expected to be well described. In order to obtain the excitation energies from the natural orbitals one needs to assign energy levels to the natural orbitals, which is a challenge of its own as the natural orbitals are not defined as the eigenstates of a Hamiltonian.

\section{MOLECULAR DISSOCIATION}

In order to investigate why RDMFT is very successful in describing molecular dissociation, we again use a 1D model system. The external potential now consists of two wells and is given by

$$
v_{\text {ext }}(x)=-\frac{v}{\cosh ^{2}(x-d / 2)}-\frac{v}{\cosh ^{2}(x+d / 2)},
$$

where $d$ describes the distance between the two wells. We choose $v=0.9$ for all the calculations in this section. The interaction remains of the form Eq. (9) with $b=0.5$. The calculations are performed in a box ranging from -20 to 20 with a grid spacing of 0.05 . The model resembles a diatomic molecule and therefore dissociates into two independent single-well fractions. It was shown that within DFT the independence of the two fragments is ensured by the appearance of a peak at the midpoint of the exact Kohn-Sham (KS) potential [20-24]. However, none of the commonly used DFT functionals reproduces the exact behavior which, at least partially, explains the failure of DFT in describing molecular dissociation. Within RDMFT the dissociation of small molecules is very well described by even the first generation functionals $[3,4]$ and further improved by the more recent ones [3]. Hence, the question arises why RDMFT is so

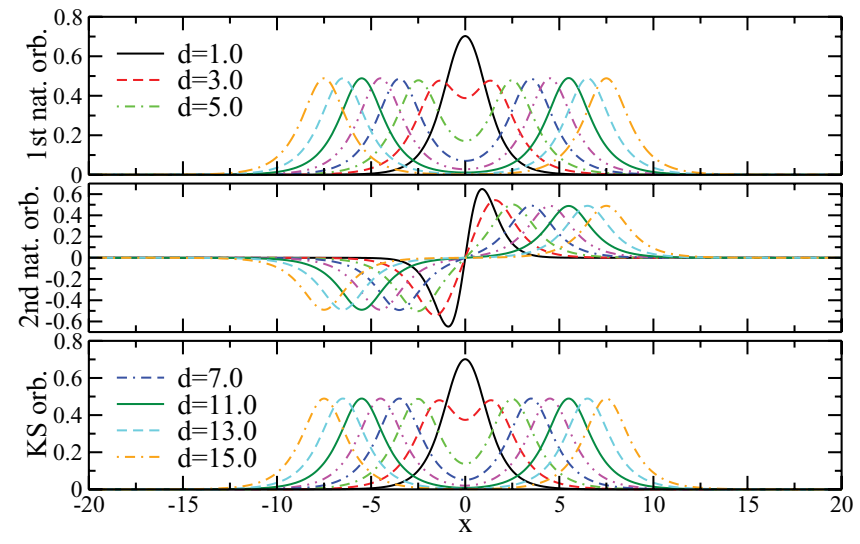

FIG. 5. (Color online) First and second natural orbital as a function of distance between the two wells. For comparison the single occupied KS orbital is also shown. (All graphs are in a.u.)

much more successful in this case. Either the natural orbitals are more suited to describe molecular dissociation than the KS orbitals or the additional freedom of fractional occupation numbers makes the difference.

Figure 5 shows the first and second natural orbitals for different distances $d$ between the wells. For comparison we also included the doubly occupied first KS orbital. We emphasize that this is the exact KS orbital which, for two electron singlet systems, is given as $\sqrt{n(\mathbf{r}) / 2}$. The occupied $\mathrm{KS}$ orbital is very similar to the first natural orbital. However, while the KS orbital is doubly occupied for all distances the occupation of the first natural orbital changes from almost two at small distances to about one for distances larger than 10 a.u., see Fig. 6. At the same time the occupation of the second natural orbital increases from zero to one such that both natural orbitals are half occupied at large distances (for closed-shell system an occupation of one corresponds to half occupation). Strictly speaking the occupation numbers of these two orbitals only become degenerate in the limit $d \rightarrow \infty$. Numerically, however, we observe that they are identical for distances larger than 10 a.u. As a result, the numerical calculation can produce

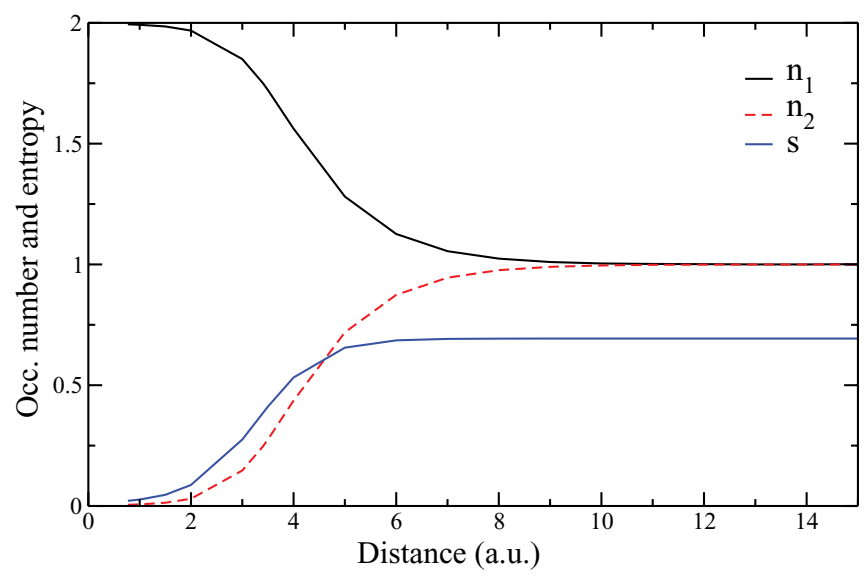

FIG. 6. (Color online) Occupation numbers and correlation entropy, s, of the ground-state density matrix as a function of distance between the two wells. 
natural orbitals that violate parity, for example if one works with an asymmetric grid. At infinite separation the degeneracy of the first two natural orbitals implies that one can use any linear combination of these orbitals without changing the 1-RDM. Especially, one can choose one natural orbital to be localized at each well mimicking the situation that appears in nature due to the small parity violating fluctuations that are present there.

Figure 6 also shows that at small distances the first natural orbital is almost fully occupied while the second is nearly empty. All other occupation numbers are too small to be visible on the scale of this figure. The fact that with increasing distance the natural orbitals become half occupied implies that the amount of correlation in the system increases with increasing distance. To quantify the correlation we calculate the correlation entropy as a function of $d$. As expected the entropy increases with increasing distance and saturates at a value of $s \approx 0.69$, the equivalent of four orbitals being occupied by half an electron, at around $d=10 \mathrm{a}$.u. the distance where the occupation numbers of the first two natural orbitals become degenerate.

\section{CONCLUSIONS}

We investigated the behavior of the natural orbitals in two different situations, a single well and two wells with different distances. It was shown that the natural orbitals of the single well system all localize if the electrons are interacting, even if the interaction strength is tiny. As a result, the ground-state natural orbitals are not a good description of an excited state if the latter is partially ionized. On the other hand, the natural orbitals of a bound excited state are rather similar to the ground-state ones such that a description of the excitation by a change in the occupation numbers alone is a good approximation. It was also shown that an increase in interaction strength leads to a partial ionization of the states which manifests itself in a partially delocalized character of the natural orbitals.

The reason RDMFT is highly successful in the description of molecular dissociation, and most likely also other strongly correlated situations, lies in the freedom of fractional occupation numbers. At all distances the first natural orbital closely resembles the occupied KS orbital of DFT. At large distances, the occupied KS orbital remains symmetric with equal contributions at each well. In contrast to that, in RDMFT it is possible to occupy two natural orbitals with the same fraction of a particle. In other words, two natural orbitals can become degenerate with respect to their occupation number and, therefore, one can perform unitary transformations in the degenerate subspace without changing the one-body density matrix. Therefore, in the dissociation limit one can choose the two natural orbitals such that they each localize at one of the fragments in resemblance of the behavior of the electrons in reality.

In the future, the problem of assigning energy levels to the natural orbitals needs to be addressed. It was shown that the natural orbitals can be obtained as the eigenstates of a single-particle Hamiltonian with a nonlocal external potential [5,25]. However, this Hamiltonian is highly nonunique. More specifically, its eigenvalues are undetermined meaning that the energy levels corresponding to the natural orbitals are arbitrary. A Hamiltonian with a local potential is not guaranteed to exist for an arbitrary set of natural orbitals but it might provide a good first approximation. Work in this direction is currently in progress.

\section{ACKNOWLEDGMENTS}

We acknowledge funding by the Spanish MEC (FIS200765702-C02-01), "Grupos Consolidados UPV/EHU del Gobierno Vasco" (IT-319-07), the European Community through e-I3 ETSF project (Contract No. 211956).
[1] P. Hohenberg and W. Kohn, Phys. Rev. 136, B864 (1964).

[2] W. Kohn and L. J. Sham, Phys. Rev. 140, A1133 (1965).

[3] O. Gritsenko, K. Pernal, and E. J. Baerends, J. Chem. Phys. 122, 204102 (2005).

[4] S. Goedecker and C. J. Umrigar, Phys. Rev. Lett. 81, 866 (1998).

[5] T. L. Gilbert, Phys. Rev. B 12, 2111 (1975).

[6] M. Piris, Int. J. Quantum Chem. 106, 1093 (2006).

[7] A. M. K. Müller, Phys. Lett. A105, 446 (1984).

[8] M. Buijse and E. J. Baerends, Mol. Phys. 100, 401 (2002).

[9] O. Leiva and M. Piris, J. Chem. Phys. 123, 214102 (2005).

[10] M. A. L. Marques and N. N. Lathiotakis, Phys. Rev. A 77, 032509 (2008).

[11] D. Rohr, K. Pernal, E. Gritsenko, and O. V. Baerends, J. Chem. Phys. 129, 164105 (2008).

[12] N. N. Lathiotakis, N. Helbig, A. Zacarias, and E. K. U. Gross, J. Chem. Phys. 130, 064109 (2009).

[13] S. Sharma, J. K. Dewhurst, N. N. Lathiotakis, and E. K. U. Gross, Phys. Rev. B 78, 201103(R) (2008).

[14] N. N. Lathiotakis, S. Sharma, J. K. Dewhurst, F. G. Eich, M. A. L. Marques, and E. K. U. Gross, Phys. Rev. A 79, 040501(R) (2009).
[15] A. J. Coleman, Rev. Mod. Phys. 35, 668 (1963).

[16] P. Ziesche, Int. J. Quantum Chem. 56, 363 (1995).

[17] M. A. L. Marques, A. Castro, G. F. Bertsch, and A. Rubio, Comput. Phys. Commun. 151, 60 (2003).

[18] A. Castro, H. Appel, M. Oliveira, C. A. Rozzi, A. Andrade, F. Lorenzen, M. A. L. Marques, E. K. U. Gross, and A. Rubio, Phys. Status Solidi B 243, 2465 (2006).

[19] L. D. Landau and E. M. Lifschitz, Quantum Mechanics (Butterworth-Heinemann, Oxford, 1977).

[20] J. P. Perdew, Density Functional Methods in Physics (Plenum Press, New York, 1985), Chap. "What do the Kohn-Sham orbital energies mean? How do atoms dissociate?", p. 265.

[21] M. A. Buijse, E. J. Baerends, and J. G. Snijders, Phys. Rev. A 40, 4190 (1989).

[22] O. V. Gritsenko, R. van Leeuwen, and E. J. Baerends, Phys. Rev. A 52, 1870 (1995).

[23] O. V. Gritsenko and E. J. Baerends, Phys. Rev. A 54, 1957 (1996).

[24] N. Helbig, I. V. Tokatly, and A. Rubio, J. Chem. Phys. 131, 224105 (2009).

[25] K. Pernal, Phys. Rev. Lett. 94, 233002 (2005). 